\title{
Die Bedeutung von Metakognitionen für das Verständnis und die Psychotherapie von Zwang
}

\author{
Angelika Neumann ${ }^{a} \quad$ Edgar Geissner ${ }^{b}$ \\ a Studienzentrum Verhaltensmedizin und Psychotherapie (SZVT), Stuttgart, \\ ${ }^{\mathrm{b}}$ Medizinisch-Psychosomatische Klinik Roseneck, Prien, und Department Psychologie, Universität München, Deutschland
}

\author{
Schlüsselwörter \\ Zwangsstörung · Metakognitionen · \\ Self-Regulatory Executive Function Modell . \\ Kognitive Verhaltenstherapie
}

\section{Zusammenfassung}

Im vorliegenden Beitrag werden drei kognitive Ansätze bei Zwangsstörungen diskutiert: (1) Die Theorie der Inhaltsspezifität von Beck, (2) der kognitiv-behaviorale Ansatz von Salkovskis, (3) die neuere Theorie der Metakognitionen von Wells. Der Ansatz von Wells wird genauer erklärt: Es werden das sogenannte "Self-Regulatory Executive Function»-Modell dargestellt sowie inhaltliche Besonderheiten des Denkens bei Zwang skizziert: der selbstreferenzielle Zustand des Denkens, Denken im Objektmodus und Aspekte der "thought-action fusion". Die Relevanz des metakognitiven Ansatzes von Wells für das Verständnis der Entstehung und Aufrechterhaltung von Zwang wird diskutiert. Darüber hinaus werden Vorschläge für die praktische Umsetzung in der Psychotherapie von Zwang abgeleitet und erläutert.

\section{Die Bedeutung von Kognitionen bei Zwang}

In den letzten 10 Jahren sind kognitive Aspekte der Zwangsstörung verstärkt in den Fokus der Forschungsbemühungen gerückt [Clark et al., 2003]. Ein wichtiger Grund, warum Kognitionen als bedeutsam für die Zwangsstörung erachtet werden, ist die Dominanz des gedanklichen Anteils an den Symptomen. Bei den meisten Zwangspatienten bestehen den Zwangshandlungen vorausgehende Zwangsgedanken bzw. Zwangsge-

\author{
Key words \\ Obsessive Compulsive Disorder - Metacognitions . \\ Self-regulatory executive function model - Cognitive \\ behavioural therapy
}

\section{Summary}

The Importance of Metacognitions in the Understanding and Treatment of Obsessive Compulsive Disorder

The present article discusses three cognitive approaches of Obsessive Compulsive Disorder (OCD): (1) Beck's theory of content-specificity, (2) Salkovskis' cognitive-behavioural approach and (3) Wells' more recent theory of metacognitions. Wells' approach is explained in more detail: the so called Self-Regulatory Executive Function model is presented as well as special aspects of thinking in OCD, for example the self-referential status of thinking, thinking in object mode and aspects of 'thought-action fusion'. The relevance of Wells' metacognitive approach for the development and the maintenance of OCD is discussed. Furthermore, proposals are made on how to include these issues in the psychotherapy of OCD.

\begin{tabular}{ll}
\hline KARGER & ( 2007 S. Karger GmbH, Freiburg \\
$\begin{array}{l}\text { Fax +4976145207 14 } \\
\begin{array}{l}\text { E-mail Information@Karger.de } \\
\text { www.karger.com }\end{array}\end{array}$ & $\begin{array}{l}\text { Accessible online at: } \\
\text { www.karger.com/ver }\end{array}$ \\
&
\end{tabular}

danken ohne Handlungen. Reinecker [2000] schätzt diesen Anteil auf 95\%. Nach dem DSM IV können Zwangsgedanken in Form von Bildern, Impulsen oder Befürchtungen auftreten [Saß et al., 1998]. Zwangsgedanken werden als aufdringlich empfunden, lösen Angst aus und veranlassen so das nachfolgende Zwangsritual. In verschiedenen Forschungsarbeiten erwiesen sich dysfunktionale Bewertungen von aufdringlichen Gedanken als zentral für die Entstehung und Aufrechterhaltung von Zwängen bzw. Subtypen von Zwangsstörungen [Ob- 
sessive Compulsive Cognitions Working Group OCCWG, 2003; Abramowitz et al., 2006]. Es bedarf jedoch dringend einer weiteren Verfeinerung der theoretischen kognitiven Modelle und Messmethoden [Taylor et al., 2002]. Im Folgenden wird nach einer Darstellung der dysfunktionalen Grundüberzeugungen nach Beck [1976] und der negativen Bewertungen nach Salkovskis [1996] die Theorie der Metakognitionen nach Wells [2000] erläutert. Abschließend wird ein integriertes Modell der Kognitionen bei Zwang vorgestellt, aus dem therapeutische Interventionen abgeleitet werden.

\section{Theorie der Inhaltsspezifität nach Beck}

Die meisten derzeit diskutierten kognitiven Modelle von Zwangsstörungen basieren auf der Theorie der Inhaltsspezifität von Beck [1976]. In seiner «Theorie der emotionalen Störungen» nimmt Beck an, dass alle affektiven Reaktionen durch die Art und Weise entstehen, wie eine Person ihre Erfahrungen interpretiert. Alle Erfahrungen seien in Form von «Schemata» im Langzeitgedächtnis gespeichert, und zwar (1) als Überzeugungen (beliefs) und (2) als Annahmen (assumptions). Überzeugungen seien als Tatsachen formuliert und würden als Wahrheit über die eigene Person und die Welt aufgefasst (Bsp.: «Ich bin ein Versager»). Annahmen seien dagegen konditional formuliert (Bsp.: «Wenn ich unbekannte Symptome verspüre, bin ich todkrank»). Bei Personen mit emotionalen Störungen fände sich nicht nur eine Vielzahl solcher dysfunktionaler Schemata, sie seien auch besonders unflexibel - anders als bei gesunden Personen, bei denen ebenfalls gelegentlich dysfunktionale Schemata zu finden seien. Außerdem geht Beck von einer sogenannten kognitiven Spezifität aus. Demnach beruhen verschiedene psychopathologische Erkrankungen auf inhaltlich unterschiedlichen dysfunktionalen Überzeugungen. Für die Entstehung von Zwangsgedanken spielten die Themen Warnung oder Zweifel eine Rolle, für Zwangshandlungen Befehle an die eigene Person, bestimmte Handlungen zu vollführen, um Gefahr abzuwenden [Beck, 1979].

\section{Kognitiv-behavioraler Ansatz nach Salkovskis}

Kernstück der kognitiv-behavioralen Theorie der Zwangserkrankung von Salkovskis [1996] ist die dysfunktionale Bewertung von aufdringlichen Gedanken. Mittlerweile gilt es als empirisch gut belegt, dass aufdringliche Gedanken, also plötzlich in das Bewusstsein einschießende und als störend empfundene Bilder, Gedanken oder Impulse bei den meisten Menschen vorkommen. Es wird eine Quote von zirka 90\% einer gesunden Population genannt. Allerdings empfinden gesunde Personen die aufdringlichen Gedanken nicht als quälend [Rachman und deSilva, 1978; Salkovskis und Harrison, 1984]. Es stellt sich die Frage, weshalb die gleichen aufdring- lichen Gedanken bei einigen Menschen persistieren und zu klinisch relevanten Zwangsstörungen führen, bei anderen hingegen wieder verschwinden. Nach Salkovskis werden aufdringliche Gedanken dann zu einem Problem, wenn sie als negativ bewertet werden. Diese Bewertung erfolge durch automatische Gedanken (Bsp.: «Es ist schlecht, einen solchen Gedanken zu haben»). Der Unterschied zu gesunden Personen ist somit (1) das Vorliegen einer dysfunktionalen Grundüberzeugung (Überschätzung der eigenen Verantwortlichkeit) und (2) die daraus resultierende automatisierte negative Bewertungen der aufdringlichen Gedanken, die unmittelbar zu einer Stimmungsverschlechterung führt (Angst, Unruhe). Diese negative affektive Reaktion führe entweder zum Versuch, den aufdringlichen Gedankens zu unterdrücken, oder zu neutralisierendem Verhalten in Form von offenen oder verdeckten Zwangsritualen. Diese gedanklichen oder Verhaltensrituale würden durch die nachfolgende Erleichterung negativ verstärkt (operantes Lernen) und somit aufrechterhalten. Bei der Entstehung der Zwangsgedanken spielt der Versuch, die aufdringlichen Gedanken zu unterdrücken, eine wesentliche Rolle. Er führt zu dem paradoxen Effekt, dass über eine verstärkte Aufmerksamkeit die Auftretenswahrscheinlichkeit dieser Gedanken erhöht wird. Zwangsrituale werden somit über mehrere Rückkopplungsschleifen aufrechterhalten und zu einem klinisch relevanten Phänomen.

\section{Die Theorie der Metakognitionen von Wells}

Wells [2000] erweiterte Salkovskis' Ansatz um eine zusätzliche Bewertungsebene, die Reflexion des eigenen Denkens allgemein. Sein Ansatz hebt sich insofern von Salkovskis' Ansatz $\mathrm{ab}$, als er eine dysfunktionale Bewertung aller Gedanken annimmt, nicht nur der Zwangsgedanken. In Weiterentwicklung des Modells von Beck betonen Wells und Matthews [1994], dass es nicht sinnvoll sei, dysfunktionale Schemata als abgeschlossene Einheiten zu sehen, die in der Therapie «ausgewechselt» werden könnten. Vielmehr müsste zunächst verstanden werden, wie eine Person Informationen verarbeitet. Es sei unwahrscheinlich, dass Überzeugungen wie «Ich bin ein Versager» wörtlich abgespeichert seien. Daher sei es notwendig zu verstehen, nach welchen Regeln eine Person ihr Denken kontrolliert, reguliert und korrigiert, bevor dieses therapeutisch verändert werden kann. Es sei also wichtiger zu verstehen, wie jemand denkt, als was er denkt. Wells und Matthews nehmen an, dass neben Überzeugungen und Annahmen bestimmte dysfunktionale Metakognitionen existieren, die für die Entstehung und Aufrechterhaltung von emotionalen Erkrankungen - somit auch von Zwangsstörungen - verantwortlich sind. Sie schließen sich dabei Flavells Verständnis an, wonach unter Metakognitionen «... jedwedes Wissen oder kognitive Prozesse, die involviert sind bei der Bewertung, Steuerung oder Kontrolle von Kognitionen» zu verstehen ist [1979; zit. nach Wells, 2000]. 


\begin{tabular}{|c|c|c|}
\hline \multicolumn{3}{|c|}{$\begin{array}{l}\text { Low Level Processing Units: situationsspezifisches, reizabhängiges } \\
\text { Denken, unbewusst, auf einer niedrigen Abstraktionsebene angesiedelt }\end{array}$} \\
\hline $\begin{array}{l}\text { «Hindurchlassen» } \\
\text { bestimmter Infos }\end{array}$ & $\downarrow \uparrow$ & $\begin{array}{l}\text { Unterdrückung oder } \\
\text { Intensivierung }\end{array}$ \\
\hline \multicolumn{3}{|c|}{$\begin{array}{l}\text { Online-Verarbeitung von Informationen, bewusste Kontrolle und } \\
\text { Bewertung von Gedanken, aktives Grübeln, Sorgen }\end{array}$} \\
\hline $\begin{array}{l}\text { Restrukturierung, } \\
\text { Elaboration }\end{array}$ & $\downarrow \uparrow$ & $\begin{array}{l}\text { Selektion eines } \\
\text { Handlungsplanes }\end{array}$ \\
\hline \multicolumn{3}{|c|}{$\begin{array}{l}\text { Self Beliefs: grundlegende metakognitive Überzeugungen über sich } \\
\text { selbst }\end{array}$} \\
\hline
\end{tabular}

Abb. 1. 3 Ebenen des Denkens nach dem S-REF-Modell von Wells und Matthews [1994].

\section{Das «Self-Regulatory Executive Function»-Modell (S-REF-Modell)}

Wells und Matthews [1994] entwickelten das sogenannte «Self-Regulatory Executive Function»-Modell (S-REF-Modell) der emotionalen Dysfunktion, in dem metakognitive Überzeugungen und Pläne eine Schlüsselrolle bei der Entstehung emotionaler Störungen spielen. Hierbei spielen gedankliche Prozesse auf drei miteinander interagierenden Abstraktionsebenen eine Rolle (Abb. 1):

Auf der ersten Ebene der Low Level Processing Units werden Informationen weitgehend unbewusst und automatisch verarbeitet. Nur einige Informationen gelangen in die bewusste Weiterverarbeitung auf der zweiten Ebene. Bei der Zwangsstörung könnten dies z.B. aufdringliche Gedanken/Zwangsgedanken sein oder andere Gedanken, die als bedeutsam erachtet werden. Entscheidend für die Entstehung emotionaler Störungen ist die zweite, metakognitive Ebene. Auf dieser Ebene werden die Gedanken, die auf der ersten Ebene «durchgelassen» werden, bewusst bewertet, kontrolliert und gesteuert (Online-Verarbeitung). Auf der dritten Ebene befinden sich die Self-Beliefs, d.h. Überzeugungen über sich selbst, das eigene Gedächtnis.

Die Online-Verarbeitung ist abhängig von den Self-Beliefs. Die Online-Verarbeitung erfolgt willentlich und ist sehr störanfällig. Hier sind die sogenannten Metakognitionen angesiedelt (Bsp.: «Wenn ich etwas denke, wird es eintreten»). Die Online-Verarbeitung wird einerseits beeinflusst von den SelfBeliefs, die vorgeben, wie mit bestimmten Gedanken umgegangen wird. Andererseits dient sie dazu, die grundlegenden Überzeugungen angepasst an die Realität abzuändern, zu restrukturieren. Bei emotionalen Störungen erfolgt keine adäquate Restrukturierung der Self-Beliefs, so dass die OnlineVerarbeitung immer auf die gleiche Weise rigide verläuft. Es kommt zu einem Teufelskreis.

\section{Charakteristika des Denkens von Zwangspatienten}

\section{Permanentes selbstreferenzielles Denken}

Im Fokus des Ansatzes von Wells und Mitarbeitern steht das selbstreferenzielle Denken, das Denken über eigene Gedanken und Gefühle. Es ist immer dann aktiviert, wenn Diskrepanzen zwischen Ist- und Soll-Zustand festgestellt werden. Bei einer gesunden Person kann diese Diskrepanz schnell reduziert werden, z.B. durch eine Änderung des Selbstbildes oder die Anpassung der Ziele. Bei einer emotionalen Störung persistiert der selbstreferenzielle Modus; Ist- und Soll-Zustand bleiben wegen fehlender oder dysfunktionaler Adaptationsmöglichkeiten diskrepant. So ist beispielsweise das Ziel «Ich muss alle Gedanken stets hundertprozentig unter Kontrolle haben» unrealistisch. Eine gesunde Person ändert dieses Ziel ab, z.B. in «Ich kann manchmal meine Gedanken kontrollieren» und beendet damit den selbstreferenziellen $\mathrm{Zu}$ stand. Ein Zwangspatient hält rigide am Ziel fest, ohne dies jemals zu erreichen, und verharrt im Denken über die eigenen Gedanken und Gefühle. Ein Charakteristikum von Zwangspatienten ist die erhöhte Aufmerksamkeit auf den eigenen Denkprozess. Das Denken ist ständig selbstreferenziell. Den Autoren zufolge richten Zwangspatienten ihre Aufmerksamkeit eher auf interne Ereignisse, insbesondere auf ungewollte automatische Gedanken. Diese interne Aufmerksamkeit reduziert das Vertrauen in das eigene Gedächtnis und führt so zum Bedürfnis nach Kontrolle. Zur Unterbrechung des Zwangsverhaltens werden dann ebenfalls sehr störanfällige interne Signale verwendet, z.B. das Gefühl der Sicherheit, perfekte Erinnerungen usw.

\section{Denken im Objektmodus}

Innerhalb des selbstreferenziellen Denkens kann das S-REFSystem auf zwei Arten aktiviert sein: im Objektmodus oder im metakognitiven Modus (Tab. 1). Im Objektmodus werden eigene Annahmen als wahr und objektiv angesehen, im metakognitiven Modus wird das Denken selbst zum Gegenstand der Betrachtung, es wird kritisch reflektiert und überprüft. Laut Wells ist der metakognitive Modus der Zustand, der Veränderung erst ermöglicht, d.h. der in der Therapie durch Interventionen hergestellt werden sollte. Zwangspatienten würden jedoch im Objektmodus denken, d.h. sie nehmen ihre Gedanken als wahr und objektiv an.

Die von Wells gewählten Begriffe «Metakognitionen» und «metakognitiver Denkmodus» sind leicht zu verwechseln. Dysfunktionale Metakognitionen sind Überzeugungen und Annahmen über das eigene Denken. Sie sind Teil des metakognitiven Wissens («metacognitive knowledge»). Metakognitionen sind bei Zwang in Form von dysfunktionalen Einstellungen vorhanden; sie tragen wesentlich zur Aufrechterhaltung der Erkrankung bei. Andererseits unterscheidet Wells verschiedene Haltungen dem eigenen Denken gegenüber (Denkmodi). Der metakognitive Denkmodus ist eine distanzierte Haltung dem eigenen Denken gegenüber. In die- 
Tab. 1. Vereinfachte Darstellung von «Objektmodus» und «metakognitivem Modus» [Wells, 2000, S. 28]

\begin{tabular}{|c|c|}
\hline Objektmodus & Metakognitiver Modus \\
\hline $\begin{array}{l}\text { Metakognitionen } \\
\text { Gedanken sind ein Spiegelbild der Realität } \\
\text { Die Befürchtungen sind real } \\
\text { Man muss nach den Gedanken handeln }\end{array}$ & $\begin{array}{l}\text { Metakognitionen } \\
\text { Gedanken sind Ereignisse, keine Realität } \\
\text { Gedanken müssen überprüft werden }\end{array}$ \\
\hline $\begin{array}{l}\text { Ziele } \\
\text { Gefahr beseitigen }\end{array}$ & $\begin{array}{l}\text { Ziele } \\
\text { Gedanken verändern }\end{array}$ \\
\hline $\begin{array}{l}\text { Strategien } \\
\text { Gefahr einschätzen } \\
\text { Gefahrreduzierendes Verhalten einsetzen } \\
\quad \text { (Sorgen, Grübeln) }\end{array}$ & $\begin{array}{l}\text { Strategien } \\
\text { Gedanken einschätzen } \\
\text { Metakognitive Kontrollstrategien ausführen: } \\
\text { Sorgen aufgeben, Aufmerksamkeit weglenken }\end{array}$ \\
\hline $\begin{array}{l}\text { Ergebnis } \\
\text { Dysfunktionale Überzeugung gefestigt }\end{array}$ & $\begin{array}{l}\text { Ergebnis } \\
\text { Wissen umstrukturiert } \\
\text { Neue Pläne entstehen }\end{array}$ \\
\hline
\end{tabular}

sem Kontext ist der metakognitive Denkmodus gesundheitsförderlich; er kommt bei Zwang kaum vor und ist in der Therapie anzustreben.

Bezüglich der Zwangsstörung nehmen die Autoren an, dass durch einen aufdringlichen Gedanken eine bestimmte dysfunktionale Metakognition aktiviert wird z.B.: «Wenn ich etwas denke, wird es passieren» (thought-event fusion). Diese Bewertung der eigenen Gedanken resultiere meist in dem Versuch, die Gedanken zu kontrollieren. Dieser wiederum führe zu der erhöhten Auftretenswahrscheinlichkeit der aufdringlichen Gedanken. Verschiedene offene und verdeckte Verhaltensweisen können erfolgen: Kontrollieren, Ordnen, Waschen, Grübeln, Zählen, Kontrolle der Gedanken und Ablenkung, Unterdrückung von Gedanken.

Weitere spezifische Denkstile von Metakognitionen bei Zwang Shafran et al. [1996] führten das Konzept der «thought-action fusion» als Kennzeichen des Denkens bei Zwang ein. Darauf aufbauend unterscheidet Wells folgende für Zwang charakteristische Denkstile:

- Thought-action fusion (TAF): Vermischung von Denken und Handeln, z.B. «Wenn ich etwas denke, werde ich es auch tun.»

- Thought-event fusion (TEF): Überzeugungen, bei denen eine Vermischung von Denken und realen Ereignissen stattfindet, z.B.: «Wenn ich etwas denke, wird es Realität.»

- Thought-object fusion (TOF): Überzeugung, dass sich die eigenen Gedanken auf Gegenstände auswirken, z.B.: «Wenn ich etwas denke, wird es auf einen Gegenstand überspringen / er wird kontaminiert werden.»

Außerdem bestehen nach Wells folgende Einstellungen zu Ritualen: (1) Positive Überzeugungen: «Wenn ich meine Rituale ausführe, bin ich sicher.»; (2) negative Überzeugungen: «Ich habe keine Kontrolle über meine Rituale.»

Über die von Wells genannten Denkstile TAF, TOF und TEF hinaus erwiesen sich verschiedene Themen als bedeutsam [Neumann, 2005]:
- Kontrolle, Perfektionismus, Verantwortung in Bezug auf eigene Gedanken/Gefühle; dazu gehören Überzeugungen wie «Ich sollte alle meine Gedanken/Gefühle unter Kontrolle haben» (Kontrolle), «Ich bin für alle meine Gedanken/Gefühle verantwortlich» (Verantwortung), «Es gibt immer einen richtigen Gedanken/immer ein richtiges Gefühl» (Perfektionismus).

- Schuld/Scham, Ängstlichkeit/Verunsicherung als Reaktion auf die eigenen Gedanken und Gefühle; hierzu gehören Einstellungen wie «Wenn ich einen negativen Gedanken habe, fühle ich mich schuldig/muss ich mich schämen» (Schuld/Scham).

- Zweifel an den eigenen kognitiven Funktionen, z.B. «Ich kann meinen Sinneswahrnehmungen/meinem Gedächtnis nicht trauen.»

Fraglich ist jedoch die Spezifität des Modells. So könnten Metakognitionen z.B. aus den Themenbereichen Verantwortung oder Schuld/Scham auch bei Depressionen relevant sein. Auch Wells selbst formulierte an anderer Stelle, dass Metakognitionen für mehrere Erkrankungen relevant seien, nämlich vermutlich für diejenigen, bei denen repetitives Denken einen wichtigen Teil der Symptomatik darstelle: Depression (Grübeln) und Zwang (Zwangsgedanken). Empirisch zu überprüfen bleibt dies außerdem für generalisierte Angststörung (Sorgen) und posttraumatische Belastungsstörung (Intrusionen).

\section{Implikationen der Theorien von Beck, Salkovskis und Wells für die Therapie der Zwangsstörung}

Nach Wells besteht ein notwendiger erster Schritt in der Therapie darin, vom Denken im Objektmodus zum Denken im metakognitiven Modus umzuschalten. Ziel in der Therapie sollte zunächst sein, die eigenen Einstellungen zu den Gedanken zu reflektieren, nicht Zwangsverhalten zu unterdrücken. Wie bei Salkovskis wird somit auch bei Wells die Bewertung 


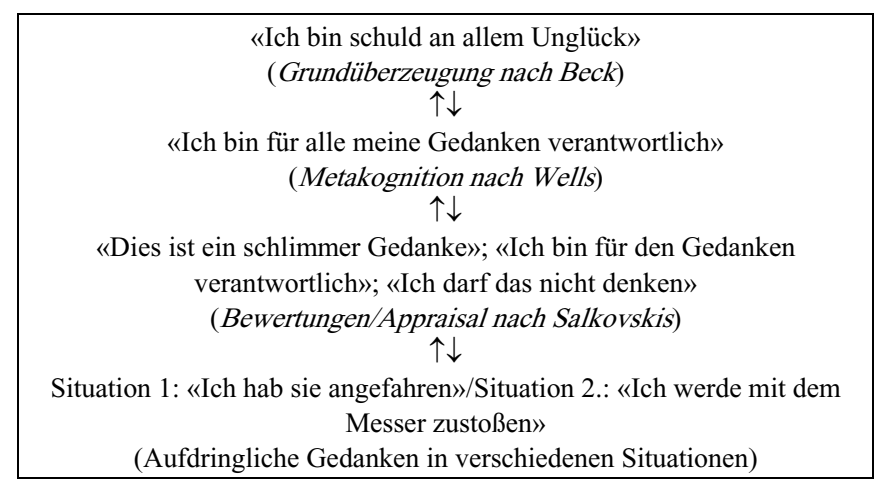

Abb. 2. Hierarchisches Modell der Kognitionen auf verschiedenen Abstraktionsebenen.

der aufdringlichen Gedanken auf einer mittleren Abstraktionsebene zum Dreh- und Angelpunkt der Theorie. Bei Wells liegt dabei der Schwerpunkt darauf, wie Zwangspatienten alle Gedanken und Gefühle reflektieren (Abb. 2). Je weiter «unten» die Gedanken im Modell (Ebene der aufdringlichen Gedanken) angesiedelt sind, desto konkreter und vielgestaltiger sind sie. Die Fülle von einzelnen Gedanken, die auf den ersten Blick nichts miteinander zu tun haben, lassen dem Therapeuten die Zwangssymptomatik oft sehr komplex erscheinen. Eine Veränderung der Kognitionen auf einer höheren Abstraktionsebene als auf der Ebene der automatischen Gedanken ermöglicht eine höhere Effektivität der Therapie. Einzelne situationsspezifische Gedanken umzuformulieren kostet mehr Zeit als eine andere Haltung oder Perspektive gegenüber den eigenen Gedanken zu vermitteln.

\section{Vorschläge für konkrete Interventionen}

\section{Allgemeine Informationsvermittlung/Psychoedukation}

Zunächst sollte dem Patienten die Theorie der Aufrechterhaltung der Zwangsstörung von Salkovskis (gut dargestellt bei Lakatos und Reinecker [1998]) vermittelt werden. Dem Patienten soll klar werden, dass die negative Bewertung der aufdringlichen Gedanken das Hauptproblem darstellt. Davon ausgehend kann das in Abbildung 2 dargestellte Modell mit Vermittlung und Betonung der metakognitiven Ebene angeführt werden. Ziel dabei ist, dem Patienten zu vermitteln, dass es metakognitive Einstellungen von größerem Allgemeinheitsgrad gibt, die den Umgang mit allen Gedanken betreffen und die zur Aufrechterhaltung der Zwangsstörung beitragen. Folgendes könnte der Therapeut dabei anführen: «In Forschungsarbeiten hat sich gezeigt, dass Zwangspatienten öfter über ihre Gedanken und ihre Gefühle nachdenken als andere Menschen. Außerdem bewerten sie ihre Gedanken negativer, versuchen sie zu steuern und zu kontrollieren. Dies trägt zur Aufrechterhaltung der Zwangserkrankung bei» [Janeck et al., 2003]. Weiterhin hilfreich kann die Vermittlung des Modells von Wells «Metakognitives Denken versus objektives Den-
Abb. 3. Beispiel für eine individuelle gedankliche Hierarchie (Patientin 1).

\begin{tabular}{l} 
Patientin 1 \\
Zwangsgedanken \\
«Ich habe mich angesteckt, ich werde meine \\
Familie anstecken, sie werden sterben, ich bin \\
schuld, das kann ich nicht ertragen ...» \\
$\uparrow \downarrow$ \\
Bewertung \\
«Das ist ein schlimmer Gedanke» \\
$\uparrow \downarrow$ \\
$\begin{array}{l}\text { Metakognition } \\
\text { «Wenn ich das denke, passiert es auch» (thought- } \\
\text { event fusion), «Ich kann meiner } \\
\text { Sinneswahrnehmung nicht trauen» (Zweifel) }\end{array}$ \\
\hline
\end{tabular}

ken» sein (Tab. 1), z.B. so: «Ein Unterschied zwischen gesunden Personen und Zwangspatienten besteht darin, dass Zwangspatienten annehmen, Gedanken seien ein Spiegelbild der Realität und keine Befürchtungen. Diese Abbildung verdeutlicht den Unterschied im Denken und soll helfen, sich von den eigenen Gedanken zu distanzieren. Wir wissen, dass dies sehr schwierig ist, insbesondere wenn durch die Befürchtungen starke unangenehme Gefühle wie Angst, Unsicherheit oder Ekel ausgelöst werden. Doch ist es unserer Erfahrung nach ein Weg, sich aus den Zwängen zu befreien.»

\section{Identifikation individueller Denkmuster}

Zunächst ist es wichtig, die individuellen Bewertungen und Metakognitionen herauszuarbeiten, z.B. folgendermaßen: «Erinnern Sie sich an einen aufdringlichen Gedanken? Wie lautet er?» und «Was denken Sie, wenn der Gedanke auftaucht? Was bedeutet dieser Gedanke für Sie? «Wie gehen Sie mit dem Gedanken um?» Die metakognitive Ebene wird dann psychoedukativ vermittelt (Abb. 3): «Viele Zwangspatienten vermischen Denken und Handeln oder Denken und Realität. Sie denken z.B.: <Wenn ich das denke, dann passiert es auch. Außerdem trauen viele Zwangspatienten ihrer eigenen Wahrnehmung nicht.» Oder auch folgendermaßen: «Sie bewerten den aufdringlichen Gedanken als negativ, weil Sie sich verantwortlich fühlen. Viele Zwangspatienten fühlen sich verantwortlich für ihre Gedanken, weil sie Denken und Handeln gleichsetzen und sie moralisch als gleichwertig empfinden.»

Die Identifikation spezifischer Metakognitionen kann auch im Zuge einer Expositionsübung erfolgen, bei der aufdringliche Gedanken und Metakognitionen erfragt werden [Fisher und Wells, 2005].

\section{Verändern dysfunktionaler Metakognitionen}

Im letzten therapeutischen Schritt sollten die dysfunktionalen Metakognitionen verändert werden. Hierbei kommen alle Techniken der kognitiven Umstrukturierung zum Einsatz. Bei einem Patienten mit der Überzeugung «Ich muss mich für negative Gedanken schämen», könnten mögliche Fragen des 
Therapeuten sein: «Welche Folgen hat das für Sie, wenn Sie so denken? Gilt das nur für Sie oder auch für andere Menschen? Was würden Sie über andere denken, wenn diese solche aufdringlichen Gedanken hätten? Was würden Sie über uns Therapeuten denken, wenn wir solche Gedanken hätten? Was würden andere über Sie denken, wenn sie wüssten, was Sie denken?» Ziel des Therapeuten sollte dabei sein, Anstöße für einen Perspektivenwechsel vom Denken im Objektmodus zum metakognitiven Modus zu geben. Wichtig ist, dass der Patient nicht zu einer veränderten Sichtweise überredet wird, sondern dass ihm selbst die Vorteile der gelassenen Haltung den eigenen Gedanken und Gefühlen gegenüber deutlich werden. Die Entscheidung, eine andere Perspektive zuzulassen, bleibt letztlich ihm selbst überlassen.

\section{Diskussion}

In diesem Beitrag wurde im Wesentlichen die relativ neue Theorie der dysfunktionalen Metakognitionen von Wells und ihre Bedeutung für Zwang dargestellt. Übergeordnete Einstellungen zu Gedanken/Gefühlen können bedeutsam für eine Verbesserung der Therapie bei Zwangsstörungen sein. Neu an diesem Ansatz ist, dass es um eine Veränderung der Gesamt- perspektive gegenüber dem eigenen Denken und Fühlen geht. Bei der Identifikation dysfunktionaler Einstellungen sollten die Themenbereiche von Wells (thought-action fusion, thought-event fusion, thought-object fusion) erweitert werden, etwa um Themen wie Verantwortung, Perfektionismus, Kontrolle, Schuld/Scham, Selbstwert oder Zweifel an den eigenen kognitiven Funktionen [OCCWG, 2003; Neumann, 2005]. Da sich die dysfunktionalen Metakognitionen zwischen verschiedenen Zwangspatienten sehr unterscheiden können, wird folgendes Vorgehen vorgeschlagen: (1) Identifikation dysfunktionaler Metakognitionen und Erstellen eines individuellen hierarchischen Kognitionsmodells, (2) Einsatz verschiedener Interventionstechniken zur Veränderung der dysfunktionalen Einstellungen mit dem Ziel größerer Gelassenheit gegenüber sogenannter aufdringlicher Gedanken.

Nach neueren Studien sind die Befunde zur therapeutischen Wirksamkeit von kognitiven Ansätzen inkonsistent, wobei z.B. spezielle Indikationen diskutiert werden und überprüft werden sollten [Abramowitz et al., 2005]. Trotzdem erscheint der metakognitive Ansatz als sinnvolle Ergänzung bisheriger Interventionen bei Zwang, zumal die Erfolgsraten bisheriger Behandlungsansätze noch immer mäßig sind [Ecker, 2005; Reinecker, 2003; Clark, 2005].

\section{Literatur}

Abramowitz JS, Taylor S, McKay D: Potentials and limitations of cognitive treatments for obsessive-compulsive disorder. Cognit Behav Ther 2005;34:140-7.

Abramowitz JS, Khandker M, Nelson CA, Deacon BJ, Rygwall R: The role of cognitive factors in the pathogenesis of obsessive-compulsive symptoms: A prospective study. Behav Res Therapy 2006;9:1361-74.

Beck AT: Cognitive Therapy and the Emotional Disorders. New York, International Universities Press, 1976. Beck AT: Wahrnehmung der Wirklichkeit und Neurose - kognitive Therapie emotionaler Störungen. München, Pfeiffer, 1979.

Clark DA: Focus on «cognition» in cognitive behavior therapy for ocd: Is it really necessary? Cognit Behav Ther 2005;34:131-9.

Clark DA, Purdon C, Wang A: The Meta-Cognitive Beliefs Questionnaire: Development of a measure of obsessional beliefs. Behav Res Ther 2003;41:655-69.

Ecker W: Therapeutische Fehler und Misserfolge in der kognitiv-behavioralen Therapie von Zwangserkrankungen aus der Perspektive der klinischen Praxis. Verhaltensther Verhaltensmed 2005;26:239-260.
Fisher PL, Wells A: Experimental modification of beliefs in obsessive-compulsive disorder: A test of the metacognitive model. Behav Res Ther 2005;43:821-9. Janeck AS, Calamari JE, Riemann BC, Heffelfinger SK: Too much thinking about thinking? Metacognitive differences in obsessive-compulsive disorder. J Anxiety Disord 2003;17:181-95.

Lakatos A, Reinecker H: Kognitive Verhaltenstherapie bei Zwängen. Psychother Psychiatr Psychother Med Klin Psychol 1998;3:201-14.

Neumann A: Metakognitionen bei Zwangsstörungen. Dissertation, Universität Bamberg 2005. www.opusbayern.de/uni-bamberg/uni/meta.php.

Obsessive Compulsive Cognitions Working Group; OCCWG (ed): Psychometric validation of the Obsessive Beliefs Questionnaire and the Interpretation of Intrusions Inventory: Part 1. Behav Res Ther 2003;41: 863-78.

Rachman SJ, deSilva S: Abnormal and normal obsessions. Behav Res Ther 1978;16:233-48.

Reinecker H: Zwangserkrankungen im verhaltenstherapeutischen Kontext; in Senf W, Broda M (Hrsg): Praxis der Paychotherapie. Ein integratives Lehrbuch. Stuttgart, Thieme, 2000.

Reinecker H: Verhaltenstherapie bei Zwangserkrankungen - Beschreibung, Klassifikation und theoretische Modelle. Psychother Dialog 2003;4:230-8.
Salkovskis PM: Cognitive-behavioural approaches to the understanding of obsessive-compulsive problems; in Rapee RM (ed): Current Controversies in the Anxiety Disorders. New York, Guilford, 1996, pp 103-133. Salkovskis PM, Harrison R: Abnormal and normal obsessions: A replication. Behav Res Ther 1984:22: 549-552.

Salkovskis PM, Warwick HM: Cognitive therapy of obsessive compulsive disorder; in Perris C, Blackburn IM, Perris H (eds): Cognitive Psychotherapy - Therory and Practice. Berlin, Springer, 1988.

Saß H, Wittchen HU, Zaudig M, Houben I: Diagnostische Kriterien DSM IV. Göttingen: Hogrefe, 1998.

Shafran R, Thordarson D, Rachman S: Thought-action fusion in obsessive-compulsive disorder. J Anxiety Disord 1996;10:379-91.

Taylor S, Kyrios M, Thordarson DS, Steketee G, Frost RO: Development and validation of instruments for measuring intrusions and beliefs in obsessive-compulsive disorder; in Frost RO, Steketee G (eds): Cognitive Approaches to Obsessions and Compulsions: Theory, Research and Treatment. Oxford, Elsevier, 2002.

Wells A: Emotional Disorders and Metacognition - Innovative Cognitive Therapy. Chichester, John Wiley, 2000.

Wells A, Matthews G: Attention and Emotion. A Clinical Perspective. Hove, Erlbaum, 1994. 